\title{
JACOBI-WEIGHTED ORTHOGONAL POLYNOMIALS ON TRIANGULAR DOMAINS
}

\author{
A. RABABAH AND M. ALQUDAH
}

Received 25 March 2004 and in revised form 20 March 2005

We construct Jacobi-weighted orthogonal polynomials $\mathscr{P}_{n, r}^{(\alpha, \beta, \gamma)}(u, v, w), \alpha, \beta, \gamma>-1, \alpha+$ $\beta+\gamma=0$, on the triangular domain $T$. We show that these polynomials $\mathscr{P}_{n, r}^{(\alpha, \beta, \gamma)}(u$, $v, w)$ over the triangular domain $T$ satisfy the following properties: $\mathscr{P}_{n, r}^{(\alpha, \beta)}(u, v, w) \in \mathscr{L}_{n}$, $n \geq 1, r=0,1, \ldots, n$, and $\mathscr{P}_{n, r}^{(\alpha, \beta, \gamma)}(u, v, w) \perp \mathscr{P}_{n, s}^{(\alpha, \beta, \gamma)}(u, v, w)$ for $r \neq s$. Hence, $\mathscr{P}_{n, r}^{(\alpha, \beta, \gamma)}(u, v, w)$, $n=0,1,2, \ldots, r=0,1, \ldots, n$, form an orthogonal system over the triangular domain $T$ with respect to the Jacobi weight function. These Jacobi-weighted orthogonal polynomials on triangular domains are given in Bernstein basis form and thus preserve many properties of the Bernstein polynomial basis.

\section{Introduction}

Recent years have seen a great deal in the field of orthogonal polynomials, a subject closely related to many important branches of analysis. Among these orthogonal polynomials, the Jacobi orthogonal polynomials are the most important. However, the cases of two or more variables of orthogonal polynomials on triangular domains have been studied by few researchers; although the main definitions and some simple properties were considered many years ago, see $[1,3,12,14]$.

Orthogonal polynomials with Jacobi weight function $w^{(\alpha, \beta, \gamma)}(u, v, w)=u^{\alpha} v^{\beta}(1-w)^{\gamma}$, $\alpha, \beta, \gamma>-1$ on triangular domain $T$ are defined in [11]. These polynomials $P_{n, r}^{(\alpha, \beta, \gamma)}(u, v, w)$ are orthogonal to each polynomial of degree less than or equal to $n-1$, with respect to the defined weight function $w^{(\alpha, \beta, \gamma)}(u, v, w)$ on $T$. However, $P_{n, r}^{(\alpha, \beta, \gamma)}(u, v, w), P_{n, s}^{(\alpha, \beta, \gamma)}(u$, $v, w), r \neq s$, are not orthogonal with respect to the weight function $w^{(\alpha, \beta, \gamma)}(u, v, w)$ on $T$.

In [5], orthogonal polynomials with respect to the weight function $w(u, v, w)=1$ on a triangular domain $T$ are defined. These polynomials $P_{n, r}(u, v, w)$ are orthogonal to each polynomial of degree less than or equal to $n-1$ and also orthogonal to each polynomial $P_{n, s}(u, v, w), r \neq s$.

In this paper, we construct orthogonal polynomials $\mathscr{P}_{n, r}^{(\alpha, \beta, \gamma)}(u, v, w)$ with respect to the Jacobi weight function $w^{(\alpha, \beta, \gamma)}(u, v, w)=u^{\alpha} v^{\beta}(1-w)^{\gamma}, \alpha, \beta, \gamma>-1, \alpha+\beta+\gamma=0$, on triangular domain $T$. These Jacobi-weighted orthogonal polynomials on triangular domains 
are given in the Bernstein basis form, and thus preserve many geometric properties of the Bernstein polynomial basis. We show that these polynomials $\mathscr{P}_{n, r}^{(\alpha, \beta, \gamma)}(u, v, w)$ over the triangular domain $T$ satisfy the following properties: $\mathscr{P}_{n, r}^{(\alpha, \beta, \gamma)}(u, v, w) \in \mathscr{L}_{n}, n \geq 1, r=$ $0,1, \ldots, n$, and for $r \neq s$ we proved that $\mathscr{P}_{n, r}^{(\alpha, \beta, \gamma)}(u, v, w) \perp \mathscr{P}_{n, s}^{(\alpha, \beta, \gamma)}(u, v, w)$. And hence, these bivariate polynomials $\mathscr{P}_{n, r}^{(\alpha, \beta, \gamma)}(u, v, w), r=0,1, \ldots, n$, and $n=0,1,2, \ldots$, form an orthogonal system over the triangular domain $T$ with respect to the weight function $w^{(\alpha, \beta, \gamma)}(u$, $v, w), \alpha, \beta, \gamma>-1, \alpha+\beta+\gamma=0$.

The construction of bivariate orthogonal polynomials on the square is straightforward. We consider the tensor product of the set of orthogonal polynomials over the square

$$
G=\{(x, y):-1 \leq x \leq 1,-1 \leq y \leq 1\}
$$

Let $\left\{P_{n}^{\left(\alpha_{1}, \beta_{1}\right)}(x)\right\}$ be the Jacobi polynomials over $[-1,1]$ with respect to the weight function $w_{1}(x)=(1-x)^{\alpha_{1}}(1+x)^{\beta_{1}}$. And let $\left\{Q_{m}^{\left(\alpha_{2}, \beta_{2}\right)}(y)\right\}$ be the Jacobi polynomials over $[-1,1]$ with respect to the weight function $w_{2}(y)=(1-y)^{\alpha_{2}}(1+y)^{\beta_{2}}$. We define the bivariate polynomials $\left\{R_{n m}(x, y)\right\}$ on $G$ formed by the tensor products of the Jacobi polynomials by

$$
R_{n m}(x, y):=P_{n-m}^{\left(\alpha_{1}, \beta_{1}\right)}(x) Q_{m}^{\left(\alpha_{2}, \beta_{2}\right)}(y), \quad n=0,1,2, \ldots, m=0,1, \ldots, n
$$

Then $\left\{R_{n m}(x, y)\right\}$ are orthogonal on the square $G$ with respect to the weight function $w(x, y)=w_{1}^{\left(\alpha_{1}, \beta_{1}\right)}(x) w_{2}^{\left(\alpha_{2}, \beta_{2}\right)}(y)$. However, the construction of orthogonal polynomials over a triangular domain is not straightforward like the tensor product over the square $G$.

For $m \geq 1$, we define the space $\mathscr{L}_{m}$ of polynomials of degree $m$ that are orthogonal to all polynomials of degree less than $m$ over a triangular domain $T$, that is,

$$
\mathscr{L}_{m}=\left\{p \in \Pi_{m}: p \perp \Pi_{m-1}\right\},
$$

and $\Pi_{n}$ is the space of all polynomials of degree $n$ over the triangular domain $T$.

This paper is organized as follows: in Section 2, we define and discuss the relation between the univariate Bernstein and Jacobi polynomials. In Sections 3 and 4, the barycentric coordinates and the generalized Bernstein polynomials over triangular domain are introduced. Properties of the orthogonal polynomials over triangular domain are given is Section 5. The construction of the Jacobi-weighted orthogonal polynomials over triangular domain with its Bernstein representation are analyzed in Sections 6 and 7.

\section{Univariate Bernstein and Jacobi polynomials}

The Bernstein polynomials $b_{i}^{n}(u), u \in[0,1], i=0,1, \ldots, n$, are defined by

$$
b_{i}^{n}(u)= \begin{cases}\left(\begin{array}{l}
n \\
i
\end{array}\right) u^{i}(1-u)^{n-i}, & i=0,1, \ldots, n \\
0 & \text { else }\end{cases}
$$


where the binomial coefficients are given by

$$
\left(\begin{array}{l}
n \\
i
\end{array}\right)= \begin{cases}\frac{n !}{i !(n-i) !} & \text { if } 0 \leq i \leq n \\
0 & \text { else. }\end{cases}
$$

The Jacobi polynomials $P_{n}^{(\alpha, \beta)}(x)$ of degree $n$ are the orthogonal polynomials, except for a constant factor, on $[-1,1]$ with respect to the weight function

$$
w(x)=(1-x)^{\alpha}(1+x)^{\beta}, \quad \alpha, \beta>-1 .
$$

In this paper, it is appropriate to take $u \in[0,1]$ for both Bernstein and Jacobi polynomials. The following two lemmas will be needed in the construction of the orthogonal bivariate polynomials and the proof of the main results.

Lemma 2.1 (see [10]). The Jacobi polynomial $P_{r}^{(\alpha, \beta)}(u)$ of degree $r$ has the following Bernstein representation

$$
P_{r}^{(\alpha, \beta)}(u)=\sum_{i=0}^{r}(-1)^{r-i} \frac{\left(\begin{array}{c}
r+\alpha \\
i
\end{array}\right)\left(\begin{array}{c}
r+\beta \\
r-i
\end{array}\right)}{\left(\begin{array}{l}
r \\
i
\end{array}\right)} b_{i}^{r}(u), \quad r=0,1, \ldots
$$

The Pochhammer symbol is more appropriate, but the combinatorial notation gives more compact and readable formulas, these have also been used by Szegö [13].

Lemma 2.2 (see [10]). The Jacobi polynomials $P_{0}^{(\alpha, \beta)}(u), \ldots, P_{n}^{(\alpha, \beta)}(u)$ of degree less than or equal to $n$ can be expressed in terms of the Bernstein basis of fixed degree $n$ by the following formula:

$$
P_{r}^{(\alpha, \beta)}(u)=\sum_{i=0}^{n} \mu_{i, r}^{n} b_{i}^{n}(u), \quad r=0,1, \ldots, n,
$$

where for $i=0, \ldots, n$,

$$
\mu_{i, r}^{n}=\left(\begin{array}{c}
n \\
i
\end{array}\right)^{-1} \sum_{k=\max (0, i+r-n)}^{\min (i, r)}(-1)^{r-k}\left(\begin{array}{c}
n-r \\
i-k
\end{array}\right)\left(\begin{array}{c}
r+\alpha \\
k
\end{array}\right)\left(\begin{array}{c}
r+\beta \\
r-k
\end{array}\right) .
$$

\section{Barycentric coordinates}

Consider a base triangle in the plane with the vertices $\mathbf{p}_{k}=\left(x_{k}, y_{k}\right), k=1,2,3$. Then every point $\mathbf{p}$ inside the triangle $T$ can be written using the barycentric coordinates $(u, v, w)$, where $u+v+w=1, u, v, w \geq 0$ as $\mathbf{p}=u \mathbf{p}_{1}+v \mathbf{p}_{2}+w \mathbf{p}_{3}$. The barycentric coordinates are the ratio of areas of subtriangles of the base triangle as follows:

$$
u=\frac{\operatorname{area}\left(\mathbf{p}, \mathbf{p}_{2}, \mathbf{p}_{3}\right)}{\operatorname{area}\left(\mathbf{p}_{1}, \mathbf{p}_{2}, \mathbf{p}_{3}\right)}, \quad v=\frac{\operatorname{area}\left(\mathbf{p}_{1}, \mathbf{p}, \mathbf{p}_{3}\right)}{\operatorname{area}\left(\mathbf{p}_{1}, \mathbf{p}_{2}, \mathbf{p}_{3}\right)}, \quad w=\frac{\operatorname{area}\left(\mathbf{p}_{1}, \mathbf{p}_{2}, \mathbf{p}\right)}{\operatorname{area}\left(\mathbf{p}_{1}, \mathbf{p}_{2}, \mathbf{p}_{3}\right)},
$$

where area $\left(\mathbf{p}_{1}, \mathbf{p}_{2}, \mathbf{p}_{3}\right) \neq 0$, which means that $\mathbf{p}_{1}, \mathbf{p}_{2}, \mathbf{p}_{3}$ are not collinear. 
208 Jacobi orthogonal polynomials on triangular domains

\section{Generalized Bernstein polynomials}

Let $T$ be a triangular domain defined by

$$
T=\{(u, v, w): u, v, w \geq 0, u+v+w=1\} .
$$

Let the notation $\alpha=(i, j, k)$ denote triples of nonnegative integers, where $|\alpha|=i+j+$ $k$. The generalized Bernstein polynomials of degree $n$ on the triangular domain $T$ are defined by the formula

$$
b_{\alpha}^{n}(u, v, w)=\left(\begin{array}{l}
n \\
\alpha
\end{array}\right) u^{i} v^{j} w^{k}, \quad|\alpha|=n,
$$

where

$$
\left(\begin{array}{l}
n \\
\alpha
\end{array}\right)=\frac{n !}{i ! j ! k !} .
$$

Note that the generalized Bernstein polynomials are nonnegative over $T$, and form a partition of unity, that is,

$$
1=(u+v+w)^{n}=\sum_{\substack{0 \leq i, j, k \leq n \\ i+j+k=n}} \frac{n !}{i ! j ! k !} u^{i} v^{j} w^{k}
$$

The sum involves a total of $(1 / 2)(n+1)(n+2)$ linearly independent polynomials. These polynomials define the Bernstein basis for the space $\Pi_{n}$ over the triangular domain $T$.

Any polynomial $P(u, v, w)$ of degree $n$ can be written in the Bernstein form

$$
P(u, v, w)=\sum_{|\alpha|=n} d_{\alpha} b_{\alpha}^{n}(u, v, w)
$$

with Bézier coefficients $d_{\alpha}$. We can also use the degree elevation algorithm for the Bernstein representation (4.5). This is obtained by multiplying both sides by $1=u+v+w$, and writing

$$
P(u, v, w)=\sum_{|\alpha|=n+1} d_{\alpha}^{(1)} b_{\alpha}^{n+1}(u, v, w)
$$

The new coefficients $d_{\alpha}^{(1)}$ are defined by, see [4, 7],

$$
d_{i j k}^{(1)}=\frac{1}{n+1}\left(i d_{i-1, j, k}+j d_{i, j-1, k}+k d_{i, j, k-1}\right), \quad i+j+k=n+1 .
$$

The Bernstein polynomials $b_{\alpha}^{n}(u, v, w),|\alpha|=n$, on $T$ satisfy, see $[5,9]$,

$$
\iint_{T} b_{\alpha}^{n}(u, v, w) d A=\frac{\Delta}{(n+1)(n+2)},
$$

where $\Delta$ is double the area of $T$. 
Let $P(u, v, w)$ and $Q(u, v, w)$ be two bivariate polynomials over $T$, then we define their inner product over $T$ by

$$
\langle P, Q\rangle=\frac{1}{\Delta} \iint_{T} P Q d A
$$

We say that $P$ and $Q$ are orthogonal if $\langle P, Q\rangle=0$.

\section{Orthogonal polynomials on triangular domain}

A basis of linearly independent and mutually orthogonal polynomials in the barycentric coordinates $(u, v, w)$ are constructed over $T$. These polynomials are represented in the following triangular table

$$
\begin{array}{rll}
P_{0,0}^{(\alpha, \beta, \gamma)}(u, v, w) & \\
P_{1,0}^{(\alpha, \beta, \gamma)}(u, v, w), & P_{1,1}^{(\alpha, \beta, \gamma)}(u, v, w) \\
P_{2,0}^{(\alpha, \beta, \gamma)}(u, v, w), & P_{2,1}^{(\alpha, \beta, \gamma)}(u, v, w), & P_{2,2}^{(\alpha, \beta, \gamma)}(u, v, w) \\
\vdots & \\
P_{n, 0}^{(\alpha, \beta, \gamma)}(u, v, w), & P_{n, 1}^{(\alpha, \beta, \gamma)}(u, v, w), & P_{n, 2}^{(\alpha, \beta, \gamma)}(u, v, w), \ldots, P_{n, n}^{(\alpha, \beta, \gamma)}(u, v, w) .
\end{array}
$$

The $k$ th row of this triangle table contains $k+1$ polynomials. Thus, for a basis of linearly independent polynomials of total degree $n$, there are $(1 / 2)(n+1)(n+2)$ polynomials.

Analogous to [5], a simple closed-form representation of degree-ordered system of orthogonal polynomials is constructed on a triangular domain $T$. Since the Bernstein polynomials are stable, see [6], it is convenient to write these polynomials in Bernstein form.

Let $f(u, v, w)$ be an integrable function over $T$, and consider the operator

$$
S_{n}(f)=(n+1)(n+2) \sum_{|\alpha|=n}\left\langle f, b_{\alpha}^{n}\right\rangle b_{\alpha}^{n} .
$$

For $n \geq m$,

$$
\lambda_{m, n}=\frac{(n+2) ! n !}{(n+m+2) !(n-m) !}
$$

is an eigenvalue of the operator $S_{n}$, and $\mathscr{L}_{m}$ is the corresponding eigenspace, see [2].

The following three lemmas will be needed in the proof of the main results.

Lemma 5.1 (see [5]). Let $P=\sum_{|\alpha|=n} c_{\alpha} b_{\alpha}^{n} \in \mathscr{L}_{m}$ and let $Q=\sum_{|\alpha|=n} d_{\alpha} b_{\alpha}^{n} \in \Pi_{n}$ with $m \leq n$. Then,

$$
\langle P, Q\rangle=\frac{(n !)^{2}}{(n+m+2) !(n-m) !} \sum_{|\alpha|=n} c_{\alpha} d_{\alpha} .
$$


210 Jacobi orthogonal polynomials on triangular domains

Lemma $5.2($ see $[5,8])$. Let $P=\sum_{|\alpha|=n} c_{\alpha} b_{\alpha}^{n} \in \Pi_{n}$. Then,

$$
P \in \mathscr{L}_{n} \Longleftrightarrow \sum_{|\alpha|=n} c_{\alpha} d_{\alpha}=0 \quad \forall Q=\sum_{|\alpha|=n} d_{\alpha} b_{\alpha}^{n} \in \Pi_{n-1}
$$

Consider the polynomials

$$
q_{n, r}(w)=\sum_{j=0}^{n-r}(-1)^{j}\left(\begin{array}{c}
n+r+1 \\
j
\end{array}\right) b_{j}^{n-r}(w) .
$$

The polynomial $q_{n, r}(w)$ is a scalar multiple of $P_{n-r}^{(0,2 r+1)}(1-2 w)$, and we have the following lemma

Lemma 5.3 (see [5]). For $r=0, \ldots, n$ and $i=0, \ldots, n-r-1, q_{n, r}(w)$ is orthogonal to $(1-$ $w)^{2 r+i+1}$ on $[0,1]$, and hence

$$
\int_{0}^{1} q_{n, r}(w) P(w)(1-w)^{2 r+1} d w=0
$$

for every polynomial $P(w)$ of degree less than or equal to $n-r-1$.

\section{Jacobi-weighted orthogonal polynomials}

For $n=0,1,2, \ldots$ and $r=0,1, \ldots, n$, we define the bivariate polynomials

$$
\mathscr{P}_{n, r}^{(\alpha, \beta, \gamma)}(u, v, w)=\sum_{i=0}^{r} c(i, \alpha, \beta) b_{i}^{r}(u, v) \sum_{j=0}^{n-r}(-1)^{j}\left(\begin{array}{c}
n+r+1 \\
j
\end{array}\right) b_{j}^{n-r}(w, u+v)
$$

where $\alpha, \beta, \gamma>-1, \alpha+\beta+\gamma=0$,

$$
\begin{gathered}
c(i, \alpha, \beta)=(-1)^{r-i} \frac{\left(\begin{array}{c}
r+\alpha \\
i
\end{array}\right)\left(\begin{array}{c}
r+\beta \\
r-i
\end{array}\right)}{\left(\begin{array}{c}
r \\
i
\end{array}\right)}, \quad i=0,1, \ldots, r, \\
b_{i}^{r}(u, v)=\left(\begin{array}{c}
r \\
i
\end{array}\right) u^{i} v^{r-i}, \quad i=0,1, \ldots, r .
\end{gathered}
$$

In this section, we show that the polynomials $\mathscr{P}_{n, r}^{(\alpha, \beta, \gamma)}(u, v, w) \in \mathscr{L}_{n}, n \geq 1, r=0,1, \ldots, n$, and $\mathscr{P}_{n, r}^{(\alpha, \beta, \gamma)} \perp \mathscr{P}_{n, s}^{(\alpha, \beta, \gamma)}$ for $r \neq s$. Thus, choosing $\mathscr{P}_{0,0}^{(\alpha, \beta, \gamma)}=1$, then the polynomials $\mathscr{P}_{n, r}^{(\alpha, \beta, \gamma)}(u$, $v, w)$ for $0 \leq r \leq n$ and $n=0,1,2, \ldots$ form a degree-ordered orthogonal sequence over $T$. 
We first rewrite these polynomials in the Jacobi polynomials form

$$
\begin{aligned}
\mathscr{P}_{n, r}^{(\alpha, \beta, \gamma)}(u, v, w)= & \sum_{i=0}^{r}(-1)^{r-i} \frac{\left(\begin{array}{c}
r+\alpha \\
i
\end{array}\right)\left(\begin{array}{c}
r+\beta \\
r-i
\end{array}\right)}{\left(\begin{array}{l}
r \\
i
\end{array}\right)} b_{i}^{r}(u, v) \\
& \times \sum_{j=0}^{n-r}(-1)^{j}\left(\begin{array}{c}
n+r+1 \\
j
\end{array}\right) b_{j}^{n-r}(w, u+v) \\
= & \sum_{i=0}^{r}(-1)^{r-i} \frac{\left(\begin{array}{c}
r+\alpha \\
i
\end{array}\right)\left(\begin{array}{c}
r+\beta \\
r-i
\end{array}\right)}{\left(\begin{array}{c}
r \\
i
\end{array}\right)} \frac{b_{i}^{r}(u, v)}{(u+v)^{r}}(1-w)^{r} \\
& \times \sum_{j=0}^{n-r}(-1)^{j}\left(\begin{array}{c}
n+r+1 \\
j
\end{array}\right) b_{j}^{n-r}(w, 1-w) .
\end{aligned}
$$

Since

$$
\frac{b_{i}^{r}(u, v)}{(u+v)^{r}}=b_{i}^{r}\left(\frac{u}{1-w}\right)
$$

and using Lemma 2.1, we get

$$
\mathscr{P}_{n, r}^{(\alpha, \beta, \gamma)}(u, v, w)=P_{r}^{(\alpha, \beta)}\left(\frac{u}{1-w}\right)(1-w)^{r} q_{n, r}(w), \quad r=0, \ldots, n,
$$

where $P_{r}^{(\alpha, \beta)}(t)$ is the univariate Jacobi polynomial of degree $r$ and $q_{n, r}(w)$ is defined in (5.6).

First, we show that the polynomials $\mathscr{P}_{n, r}^{(\alpha, \beta, \gamma)}(u, v, w), r=0, \ldots, n$, are orthogonal to all polynomials of degree less than $n$ over the triangular domain $T$.

Theorem 6.1. For each $n=1,2, \ldots, r=0,1, \ldots, n$, and the weight function $w^{(\alpha, \beta, \gamma)}(u, v$, $w)=u^{\alpha} v^{\beta}(1-w)^{\gamma}$ such that $\alpha, \beta, \gamma>-1, \alpha+\beta+\gamma=0, \mathscr{P}_{n, r}^{(\alpha, \beta)}(u, v, w) \in \mathscr{L}_{n}$ holds.

Proof. For each $m=0, \ldots, n-1$, and $s=0, \ldots, m$, we construct the set of bivariate polynomials

$$
Q_{s, m}^{(\alpha, \beta)}(u, v, w)=P_{s}^{(\alpha, \beta)}\left(\frac{u}{1-w}\right)(1-w)^{m} w^{n-m-1}, \quad m=0, \ldots, n-1, s=0, \ldots, m .
$$

The span of these polynomials includes the set of Bernstein polynomials

$$
b_{j}^{m}\left(\frac{u}{1-w}\right)(1-w)^{m} w^{n-m-1}=b_{j}^{m}(u, v) w^{n-m-1}, \quad m=0, \ldots, n-1, j=0, \ldots, m,
$$

which span the space $\Pi_{n-1}$. Thus, it is sufficient to show that for each $m=0, \ldots, n-1$, $s=0, \ldots, m$, we have

$$
I:=\iint_{T} \mathscr{P}_{n, r}^{(\alpha, \beta, \gamma)}(u, v, w) Q_{s, m}^{(\alpha, \beta)}(u, v, w) w^{(\alpha, \beta, \gamma)}(u, v, w) d A=0 .
$$


212 Jacobi orthogonal polynomials on triangular domains

This is simplified to

$$
I=\Delta \int_{0}^{1} \int_{0}^{1-w} P_{r}^{(\alpha, \beta)}\left(\frac{u}{1-w}\right) q_{n, r}(w) P_{s}^{(\alpha, \beta)}\left(\frac{u}{1-w}\right) w^{n-m-1} u^{\alpha} v^{\beta}(1-w)^{\gamma+r+m} d u d w .
$$

By making the substitution $t=u /(1-w)$, we get

$$
w^{(\alpha, \beta, \gamma)}(u, v, w)=u^{\alpha} v^{\beta}(1-w)^{\gamma}=t^{\alpha}(1-t)^{\beta}(1-w)^{\alpha+\beta+\gamma} .
$$

And thus, we have

$$
\begin{aligned}
I & =\Delta \int_{0}^{1} \int_{0}^{1} P_{r}^{(\alpha, \beta)}(t) q_{n, r}(w) P_{s}^{(\alpha, \beta)}(t)(1-w)^{\alpha+\beta+\gamma+r+m+1} w^{n-m-1} t^{\alpha}(1-t)^{\beta} d t d w \\
& =\Delta \int_{0}^{1} P_{r}^{(\alpha, \beta)}(t) P_{s}^{(\alpha, \beta)}(t) t^{\alpha}(1-t)^{\beta} d t \int_{0}^{1} q_{n, r}(w)(1-w)^{\alpha+\beta+\gamma+r+m+1} w^{n-m-1} d w .
\end{aligned}
$$

If $m<r$, then we have $s<r$, and the first integral is zero by the orthogonality property of the Jacobi polynomials. If $r \leq m \leq n-1$, we have by Lemma 5.3 the second integral equal to zero. And thus the theorem follows.

Note that taking $w^{(\alpha, \beta, \gamma)}(u, v, w)=u^{\alpha} v^{\beta}(1-w)^{\gamma}$ enables us to separate the integrand in the proof of Theorem 6.1. Also note that taking $\alpha+\beta+\gamma=0$ enables us to use Lemma 5.3 in the proof of Theorem 6.1.

In the following theorem, we show that $\mathscr{P}_{n, r}^{(\alpha, \beta, \gamma)}(u, v, w)$ is orthogonal to each polynomial of degree $n$. And thus the bivariate polynomials $\mathscr{P}_{n, r}^{(\alpha, \beta, \gamma)}(u, v, w), r=0,1, \ldots, n$, and $n=0,1,2, \ldots$ form an orthogonal system over the triangular domain $T$ with respect to the weight function $w^{(\alpha, \beta, \gamma)}(u, v, w), \alpha, \beta, \gamma>-1$.

Theorem 6.2. For $r \neq s, \mathscr{P}_{n, r}^{(\alpha, \beta, \gamma)}(u, v, w) \perp \mathscr{P}_{n, s}^{(\alpha, \beta, \gamma)}(u, v, w)$ with respect to the weight function $w^{(\alpha, \beta, \gamma)}(u, v, w)=u^{\alpha} v^{\beta}(1-w)^{\gamma}$ such that $\alpha, \beta, \gamma>-1$.

Proof. For $r \neq s$, we have

$$
\begin{aligned}
I & :=\iint_{T} \mathscr{P}_{n, r}^{(\alpha, \beta, \gamma)}(u, v, w) \mathscr{P}_{n, s}^{(\alpha, \beta, \gamma)}(u, v, w) w^{(\alpha, \beta, \gamma)}(u, v, w) d A \\
& =\Delta \int_{0}^{1} \int_{0}^{1-w} P_{r}^{(\alpha, \beta)}\left(\frac{u}{1-w}\right) P_{s}^{(\alpha, \beta)}\left(\frac{u}{1-w}\right)(1-w)^{r+s} q_{n, r}(w) q_{n, s}(w) w^{(\alpha, \beta, \gamma)}(u, v, w) d u d w
\end{aligned}
$$

By making the substitution $t=u /(1-w)$, we get $w^{(\alpha, \beta, \gamma)}(u, v, w)=t^{\alpha}(1-t)^{\beta}(1-w)^{\alpha+\beta+\gamma}$. And thus, we have

$$
I=\Delta \int_{0}^{1} P_{r}^{(\alpha, \beta)}(t) P_{s}^{(\alpha, \beta)}(t) t^{\alpha}(1-t)^{\beta} d t \int_{0}^{1} q_{n, r}(w) q_{n, s}(w)(1-w)^{\alpha+\beta+\gamma+r+s+1} d w,
$$

where the first integral equals zero by the orthogonality property of the Jacobi polynomials for $r \neq s$, and thus the theorem follows. 


\section{Orthogonal polynomials in Bernstein basis}

The Bernstein-Bézier form of curves and surfaces exhibits some interesting geometric properties, see $[4,7]$. So, we write the orthogonal polynomials $\mathscr{P}_{n, r}^{(\alpha, \beta, \gamma)}(u, v, w), r=0,1$, $\ldots, n$ and $n=0,1,2, \ldots$ in the following Bernstein-Bézier form:

$$
\mathscr{P}_{n, r}^{(\alpha, \beta, \gamma)}(u, v, w)=\sum_{|\alpha|=n} a_{\alpha}^{n, r} b_{\alpha}^{n}(u, v, w) .
$$

We are interested in finding a closed form for the computation of the Bernstein coefficients $a_{\alpha}^{n, r}$. These are given explicitly in the following theorem.

THeOREM 7.1. The Bernstein coefficients $a_{\alpha}^{n, r}$ of (7.1) are given explicitly by

$$
a_{i j k}^{n, r}= \begin{cases}(-1)^{k} \frac{\left(\begin{array}{c}
n+r+1 \\
k
\end{array}\right)\left(\begin{array}{c}
n-r \\
k
\end{array}\right)}{\left(\begin{array}{l}
n \\
k
\end{array}\right)} \mu_{i, r}^{n-k}, & 0 \leq k \leq n-r, \\
0, & k>n-r,\end{cases}
$$

where $\mu_{i, r}^{n-k}$ are given in (2.6).

Proof. From (6.1), it is clear that $\mathscr{P}_{n, r}^{(\alpha, \beta, \gamma)}(u, v, w)$ has degree $\leq n-r$ in the variable $w$, and thus

$$
a_{i j k}^{n, r}=0 \quad \text { for } k>n-r .
$$

For $0 \leq k \leq n-r$, the remaining coefficients are determined by equating (6.1) and (7.1) as follows:

$$
\begin{aligned}
\sum_{i+j=n-k} a_{i j k}^{n, r} b_{i j k}^{n}(u, v, w)= & (-1)^{k}\left(\begin{array}{c}
n+r+1 \\
k
\end{array}\right) b_{k}^{n-r}(w, u+v) \\
& \times \sum_{i=0}^{r}(-1)^{r-i} \frac{\left(\begin{array}{c}
r+\alpha \\
i
\end{array}\right)\left(\begin{array}{c}
r+\beta \\
r-i
\end{array}\right)}{\left(\begin{array}{c}
r \\
i
\end{array}\right)} b_{i}^{r}(u, v) .
\end{aligned}
$$

Comparing powers of $w$ on both sides, we have

$$
\begin{aligned}
\sum_{i=0}^{n-k} a_{i j k}^{n, r} \frac{n !}{i ! j ! k !} u^{i} v^{j}= & (-1)^{k}\left(\begin{array}{c}
n+r+1 \\
k
\end{array}\right)\left(\begin{array}{c}
n-r \\
k
\end{array}\right)(u+v)^{n-r-k} \\
& \times \sum_{i=0}^{r}(-1)^{r-i} \frac{\left(\begin{array}{c}
r+\alpha \\
i
\end{array}\right)\left(\begin{array}{c}
r+\beta \\
r-i
\end{array}\right)}{\left(\begin{array}{l}
r \\
i
\end{array}\right)} b_{i}^{r}(u, v) .
\end{aligned}
$$


214 Jacobi orthogonal polynomials on triangular domains

The left-hand side of the last equation can be written in the form

$$
\begin{aligned}
\sum_{i=0}^{n-k} a_{i j k}^{n, r} \frac{n !}{i ! j ! k !} u^{i} v^{j} & =\sum_{i=0}^{n-k} a_{i j k}^{n, r} \frac{n !(n-k) !}{i ! j ! k !(n-k) !} u^{i} v^{j} \\
& =\sum_{i=0}^{n-k} a_{i j k}^{n, r} \frac{n !(n-k) !}{i !(n-k-i) ! k !(n-k) !} u^{i} v^{j} \\
& =\sum_{i=0}^{n-k} a_{i j k}^{n, r}\left(\begin{array}{l}
n \\
k
\end{array}\right) b_{i}^{n-k}(u, v) .
\end{aligned}
$$

Now, we get

$$
\begin{aligned}
\sum_{i=0}^{n-k} a_{i j k}^{n, r}\left(\begin{array}{l}
n \\
k
\end{array}\right) b_{i}^{n-k}(u, v)= & (-1)^{k}\left(\begin{array}{c}
n+r+1 \\
k
\end{array}\right)\left(\begin{array}{c}
n-r \\
k
\end{array}\right)(u+v)^{n-r-k} \\
& \times \sum_{i=0}^{r}(-1)^{r-i} \frac{\left(\begin{array}{c}
r+\alpha \\
i
\end{array}\right)\left(\begin{array}{c}
r+\beta \\
r-i
\end{array}\right)}{\left(\begin{array}{l}
r \\
i
\end{array}\right)} b_{i}^{r}(u, v) .
\end{aligned}
$$

With some binomial simplifications, and using Lemma 2.2, we get

$$
\sum_{i=0}^{n-k} a_{i j k}^{n, r}\left(\begin{array}{l}
n \\
k
\end{array}\right) b_{i}^{n-k}(u, v)=(-1)^{k}\left(\begin{array}{c}
n+r+1 \\
k
\end{array}\right)\left(\begin{array}{c}
n-r \\
k
\end{array}\right) \sum_{i=0}^{r} \mu_{i, r}^{n-k} b_{i}^{n-k}(u, v),
$$

where $\mu_{i, r}^{n-k}$ are the coefficients resulting from writing Jacobi polynomial of degree $r$ in the Bernstein basis of degree $n-k$, as defined by expression (2.6). Thus, the required Bernstein-Bézier coefficients are given by

$$
a_{i j k}^{n, r}= \begin{cases}(-1)^{k} \frac{\left(\begin{array}{c}
n+r+1 \\
k
\end{array}\right)\left(\begin{array}{c}
n-r \\
k
\end{array}\right)}{\left(\begin{array}{l}
n \\
k
\end{array}\right)} \mu_{i, r}^{n-k}, & 0 \leq k \leq n-r, \\
0, & k>n-r,\end{cases}
$$

which completes the proof of the theorem. 
To derive a recurrence relation for the coefficients $a_{i j k}^{n, r}$ of $\mathscr{P}_{n, r}^{(\alpha, \beta, \gamma)}(u, v, w)$, we consider the generalized Bernstein polynomial of degree $n-1$

$$
\begin{aligned}
b_{i j k}^{n-1}(u, v, w) & =\frac{(n-1) !}{i ! j ! k !} u^{i} v^{j} w^{k} \\
& =\frac{(n-1) !}{i ! j ! k !} u^{i} v^{j} w^{k}(u+v+w) \\
& =\frac{(i+1) n !}{n(i+1) ! j ! k !} u^{i+1} v^{j} w^{k}+\frac{(j+1) n !}{n(i !)(j+1) ! k !} u^{i} v^{j+1} w^{k}+\frac{(k+1) n !}{n(i !)(j !)(k+1) !} u^{i} v^{j} w^{k+1} \\
& =\frac{(i+1)}{n} b_{i+1, j, k}^{n}(u, v, w)+\frac{(j+1)}{n} b_{i, j+1, k}^{n}(u, v, w)+\frac{(k+1)}{n} b_{i, j, k+1}^{n}(u, v, w) .
\end{aligned}
$$

By construction of $\mathscr{P}_{n, r}^{(\alpha, \beta, \gamma)}(u, v, w)$, we have

$$
\left\langle b_{i j k}^{n-1}(u, v, w), \mathscr{P}_{n, r}^{(\alpha, \beta, \gamma)}(u, v, w)\right\rangle=0, \quad i+j+k=n-1 .
$$

Thus by Lemma 5.2, we have

$$
(i+1) a_{i+1, j, k}^{n, r}+(j+1) a_{i, j+1, k}^{n, r}+(k+1) a_{i, j, k+1}^{n, r}=0,
$$

and since we know from Theorem 7.1 that

$$
a_{i, n-i, 0}^{n, r}=\mu_{i, r}^{n} \quad \text { for } i=0,1, \ldots, n \text {, }
$$

we can use (7.12) to generate $a_{i, j, k}^{n, r}$ recursively on $k$.

\section{Closure}

We have constructed Jacobi-weighted orthogonal polynomials $\mathscr{P}_{n, r}^{(\alpha, \beta, \gamma)}(u, v, w), \alpha, \beta, \gamma \geq$ $-1, \alpha+\beta+\gamma=0$ on the triangular domain T. Since the Bernstein polynomials are stable, see [6], we write these polynomials in Bernstein basis form. The polynomials $\mathscr{P}_{n, r}^{(\alpha, \beta, \gamma)}(u, v$, $w) \in \mathscr{L}_{n}, n \geq 1, r=0,1, \ldots, n$, and $\mathscr{P}_{n, r}^{(\alpha, \beta, \gamma)}(u, v, w) \perp \mathscr{P}_{n, s}^{(\alpha, \beta, \gamma)}(u, v, w)$ for $r \neq s$. And hence, these bivariate polynomials form an orthogonal system over the triangular domain $T$ with respect to the above weight function. 
216 Jacobi orthogonal polynomials on triangular domains

The $d$-dimensional unit simplex in barycentric coordinates is defined by

$$
T_{d}=\left\{u=\left(u_{0}, \ldots, u_{d}\right): u_{j} \geq 0, \sum_{j=0}^{d} u_{j}=1\right\} .
$$

The Bernstein basis for polynomials of degree $n$ over $T_{d}$ are defined by

$$
b_{\alpha}^{n}(u)=\left(\begin{array}{l}
n \\
\alpha
\end{array}\right) u^{\alpha}=\frac{n !}{\alpha_{0} ! \cdots \alpha_{d} !} u_{0}^{\alpha_{0}} \cdots u_{d}^{\alpha_{d}}, \quad|\alpha|=n,
$$

where $\alpha=\left(\alpha_{0}, \ldots, \alpha_{d}\right) \in N_{0}^{d+1}$ and $|\alpha|=\alpha_{0}+\cdots+\alpha_{d}=n$.

The method of construction in this paper can be generalized to the case of multivariate polynomials over a $d$-dimensional simplex in any number of variables $d$.

\section{Acknowledgment}

The authors are thankful to the referee for the review, leading to a better presentation of the paper.

\section{References}

[1] S. Cooper and S. Waldron, The diagonalisation of the multivariate Bernstein operator, J. Approx. Theory 117 (2002), no. 1, 103-131.

[2] M.-M. Derriennic, On multivariate approximation by Bernstein-type polynomials, J. Approx. Theory 45 (1985), no. 2, 155-166.

[3] C. F. Dunkl and Y. Xu, Orthogonal Polynomials of Several Variables, Encyclopedia of Mathematics and its Applications, vol. 81, Cambridge University Press, Cambridge, 2001.

[4] G. Farin, Curves and Surface for Computer Aided Geometric Design, 3rd ed., Computer Science and Scientific Computing, Academic Press, Massachusetts, 1993.

[5] R. T. Farouki, T. N. T. Goodman, and T. Sauer, Construction of orthogonal bases for polynomials in Bernstein form on triangular and simplex domains, Comput. Aided Geom. Design 20 (2003), no. 4, 209-230.

[6] R. T. Farouki and V. T. Rajan, On the numerical condition of polynomials in Bernstein form, Comput. Aided Geom. Design 4 (1987), no. 3, 191-216.

[7] J. Hoschek and D. Lasser, Fundamentals of Computer Aided Geometric Design, A K Peters, Massachusetts, 1993.

[8] J. Peters and U. Reif, Least squares approximation of Bézier coefficients provides best degree reduction in the $L_{2}$-norm, J. Approx. Theory 104 (2000), no. 1, 90-97.

[9] A. Rababah, Distance for degree raising and reduction of triangular Bézier surfaces, J. Comput. Appl. Math. 158 (2003), no. 2, 233-241.

[10] , Jacobi-Bernstein basis transformation, Comput. Methods Appl. Math. 4 (2004), no. 2, 206-214.

[11] T. Sauer, The genuine Bernstein-Durrmeyer operator on a simplex, Results Math. 26 (1994), no. 1-2, 99-130.

[12] P. K. Suetin, Orthogonal Polynomials in Two Variables, Nauka, Moscow, 1999.

[13] G. Szegö, Orthogonal Polynomials, 4th ed., American Mathematical Society, Rhode Island, 1975. 
[14] S. Waldron, A generalised beta integral and the limit of the Bernstein-Durrmeyer operator with Jacobi weights, J. Approx. Theory 122 (2003), no. 1, 141-150.

A. Rababah: Department of Mathematics and Statistics, Jordan University of Science and Technology, Irbid 22110, Jordan

E-mail address: rababah@just.edu.jo

M. Alqudah: Department of Mathematics and Statistics, Jordan University of Science and Technology, Irbid 22110, Jordan

E-mail address: alqud1ma@cmich.edu 


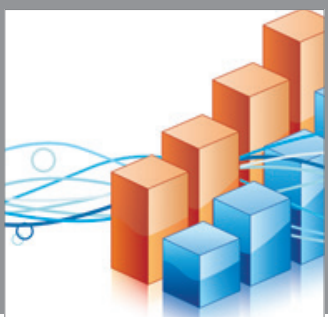

Advances in

Operations Research

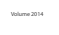

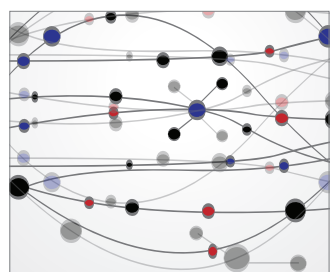

\section{The Scientific} World Journal
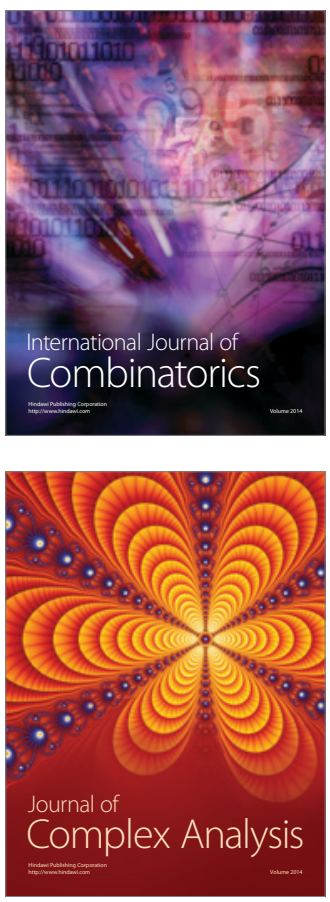

International Journal of

Mathematics and

Mathematical

Sciences
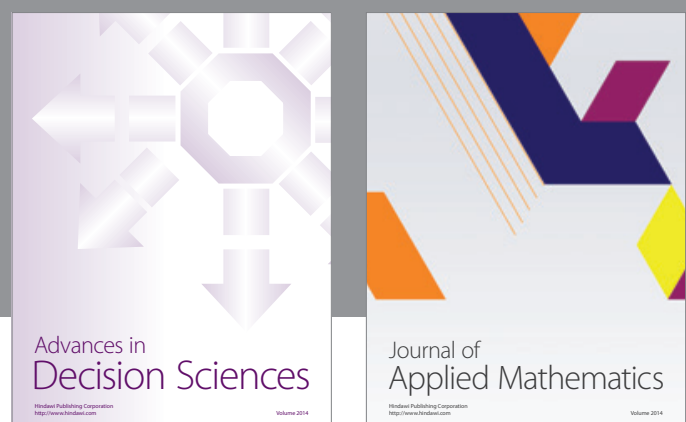

Journal of

Applied Mathematics
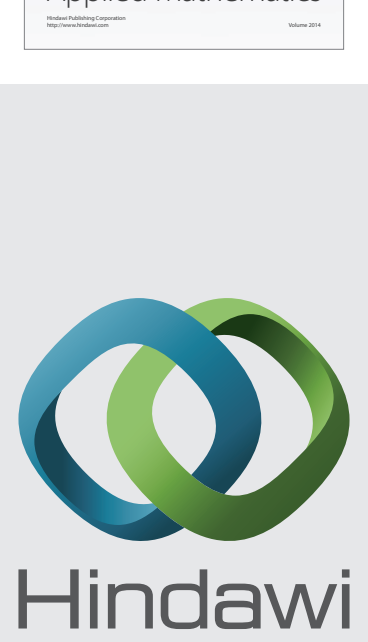

Submit your manuscripts at http://www.hindawi.com
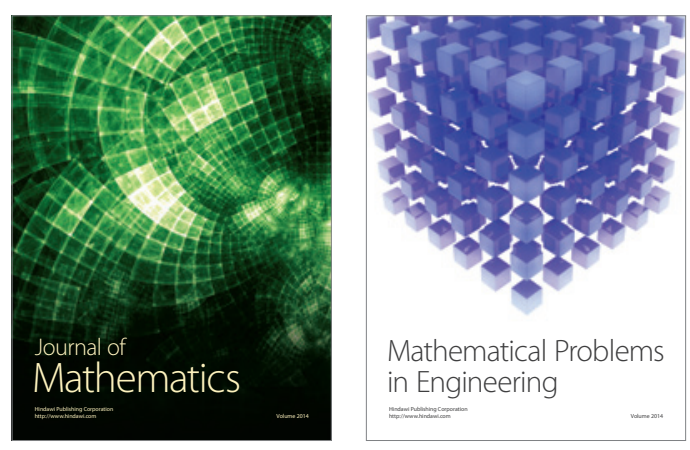

Mathematical Problems in Engineering
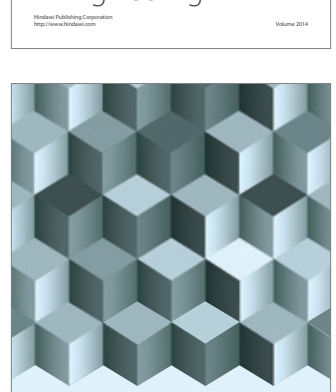

Journal of

Function Spaces
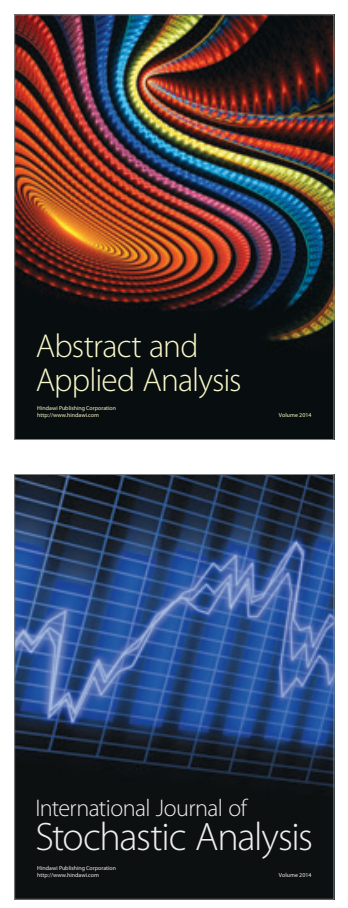

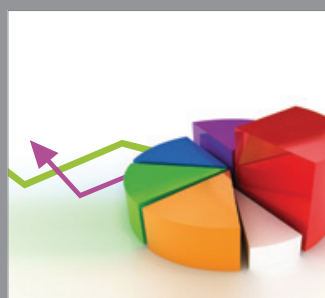

ournal of

Probability and Statistics

Promensencen
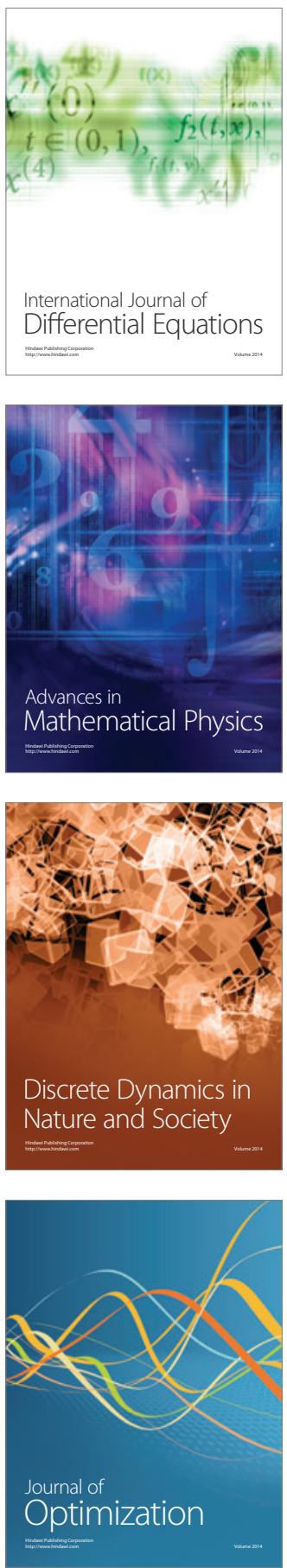